\title{
Identification and Functional Analysis of a Flavonol Synthase Gene from Grape Hyacinth
}

\author{
Hongli Liu 1,2,3, Beibei Su 1,2,3, Han Zhang 1,2,3, Jiaxin Gong 1,2,3, Boxiao Zhang 1,2,3, \\ Yali Liu 1,2,3,* and Lingjuan Du 1,2,3,* \\ 1 College of Landscape Architecture and Arts, Northwest A\&F University, Yangling 712100, Shaanxi, China; \\ liuhongli1221@sina.com (H.L.); subeibei1106@163.com (B.S.); hange@nwafu.edu.cn (H.Z.); \\ gongjiaxin@nwafu.edu.cn (J.G.); zhangboxiao@nwafu.edu.cn (B.Z.) \\ 2 State Key Laboratory of Crop Stress Biology for Arid Areas, Northwest A\&F University, Yangling 712100, \\ Shaanxi, China \\ 3 Key Laboratory of Biology and Genetic Improvement of Horticultural Crops (Northwest Region), \\ Ministry of Agriculture, Yangling 712100, Shaanxi, China \\ * Correspondence: ly16151@126.com (Y.L.); dulingjuan1980@gmail.com (L.D.); Tel.: +86-029-8708-2522 (Y.L.)
}

Received: 31 March 2019; Accepted: 19 April 2019; Published: 22 April 2019

\begin{abstract}
Flavonols are important copigments that affect flower petal coloration. Flavonol synthase (FLS) catalyzes the conversion of dihydroflavonols to flavonols. In this study, we identified a FLS gene, MaFLS, expressed in petals of the ornamental monocot Muscari aucheri (grape hyacinth) and analyzed its spatial and temporal expression patterns. qRT-PCR analysis showed that MaFLS was predominantly expressed in the early stages of flower development. We next analyzed the in planta functions of MaFLS. Heterologous expression of MaFLS in Nicotiana tabacum (tobacco) resulted in a reduction in pigmentation in the petals, substantially inhibiting the expression of endogenous tobacco genes involved in anthocyanin biosynthesis (i.e., NtDFR, NtANS, and NtAN2) and upregulating the expression of NtFLS. The total anthocyanin content in the petals of the transformed tobacco plants was dramatically reduced, whereas the total flavonol content was increased. Our study suggests that MaFLS plays a key role in flavonol biosynthesis and flower coloration in grape hyacinth. Moreover, MaFLS may represent a new potential gene for molecular breeding of flower color modification and provide a basis for analyzing the effects of copigmentation on flower coloration in grape hyacinth.
\end{abstract}

Keywords: copigmentation; flavonol; flower color; FLS; grape hyacinth

\section{Introduction}

Flower color is a key characteristic of ornamental plants [1]. Anthocyanins are flavonoids that contribute to the pink, red, orange, scarlet, violet, blue, and yellow pigmentation of ornamental plant flowers [2]. Copigmentation with other flavonoids influences the hue and intensity of flower color. Copigmentation can stabilize colored pigments and induce a blue shift in the final visible color [3]. Flavonols are one group of flavonoid that can act as copigments sandwiched between anthocyanins, causing color shifts and an increased color variety [1]. Furthermore, flavonols can themselves impart pale yellow or yellow coloration [4].

Both anthocyanins and flavonols are produced by branches of the flavonoid pathway and are derived from dihydroflavonols (dihydrokaempferol: DHK, dihydroquercetin: DHQ, and dihydromyricetin: DHM). Anthocyanins are synthesized by the sequential reaction of dihydroflavonol 4-reductase (DFR), anthocyanidin synthase (ANS), and glucosyltransferase (UFGT). In competition with DFR at a crucial branch point, individually acting FLS can catalyze the oxidation of dihydroflavonols to flavonols [4]. FLS is characterized as a soluble 2-ODD that requires cofactors 2-oxoglutarate, ferrous 
iron (II), and ascorbate and introduces the double bond between C-2 and C-3 of the C-ring [5,6]. Besides FLS, other 2-ODD enzymes, namely, flavanone $3 \beta$-hydroxylase (F3H), flavone synthase I (FNS I), and ANS, also function in flavonoid biosynthesis [7].

The first FLS cDNA was cloned from petunia (Petunia hybrida) and was functionally expressed in yeast and plants [6]. Additional FLS genes have since been identified and characterized in various plant species, such as Arabidopsis thaliana [8-10], Eustoma grandiflorum (lisianthus) [1], Citrus unshiu [11], Glycine max [12], Gentiana triflora [13], Ginkgo biloba [14], Zea mays (maize) [15], Camellia nitidissima [4], Vaccinium corymbosum [16], Allium cepa (onion) [17], Cyclamen purpurascens (cyclamen) [18], and Litchi chinensis [19]. There have been some reports of FLS genes in ornamental plants that function in flower coloration. Holton et al. demonstrated that antisense expression of a FLS gene in petunia reduced flavonol synthesis and changed the light pink coloration of petals and filaments to red. Similarly, constitutive overexpression of a FLS gene from lisianthus produced flowers with a deeper magenta coloration than the wild type (WT) plants [1]. Heterologous expression of CnFLS1 in Nicotiana tabacum (tobacco) changed floral coloration, resulting in white flowers [4]. Akita et al. isolated two FLS genes (CpurFLS1 and CpurFLS2) and discussed their involvement in flower coloration in cyclamen [18]. Furthermore, previous studies also showed that FLS can cooperate with DFR to control the metabolic balance between the anthocyanin and flavonol branches of the flavonoid pathway to ultimately determine the formation of flower color [20], or identified the transcription factor R2R3-MYB, which regulates FLS to affect spatial pattern variation of floral pigments [21].

Grape hyacinth (Muscari spp.), named for its unique grape-like color and shape, is a monocotyledonous ornamental bulbous plant that blossoms in mid-spring [22,23]. Generally, grape hyacinth varieties display a single color, such as white, pink, purple, azure, cobalt, violet, or lavender. Rarely, some species produce upper flowers that are a different color to the lower flowers [22]. Because of its variable flower coloration, grape hyacinth is a good model to study the secondary metabolism of flower coloration in monocots [22,24]. Previous studies of grape hyacinth flower coloration have focused on the chemical basis of anthocyanin-related pigmentation and on the functions of structural and regulatory proteins involved in anthocyanin biosynthesis $[22,23,25]$. Few studies have examined the compounds involved in copigmentation or analyzed the genes affecting copigmentation. It is valuable to elucidate the mechanism of flower color formation or create the novel color morphs of grape hyacinth via molecular regulation of the key gene ( $F L S)$ for flavonol synthesis.

In this study, we identified a FLS gene expressed in grape hyacinth petals, MaFLS, and analyzed its spatial and temporal expression patterns. Moreover, we measured flavonol contents at different floral developmental stages and in different organs. Via heterologous expression in tobacco, we examined the function of MaFLS in planta by examining the effect on phenotype, flavonoid content, and expression of endogenous genes in the petals of the transgenic tobacco lines. We discuss the potential role of MaFLS during floral coloration of grape hyacinth. Overall, our study provides further evidence to a growing literature on FLS-DFR competition and provides the basis for analyzing the effects of copigmentation on flower coloration in grape hyacinth.

\section{Results}

\subsection{MaFLS Gene Cloning and Sequence Analysis}

We searched for FLS homologs in the transcriptome of $M$. armeniacum flowers [24] by local BlastP querying with the characterized A. thaliana FLS1 $[9,26,27]$. One FLS homolog was identified and designated MaFLS (GenBank accession number MH636605) and its cDNA was isolated by RACE-PCR and PCR. MaFLS consisted of 1418 nucleotides with a poly(A) tail, of which 993 bp represented an ORF encoding 330 amino acid residues, with a molecular weight of $36.45 \mathrm{kDa}$ and a theoretical pI of 6.33 .

Comparison of the deduced MaFLS amino acid sequence and that of other FLS proteins with known functions revealed that it shared $72 \%$ and $71 \%$ identity with monocot Allium cepa AcFLS-HRB (AY647262) and AcFLS-H6 (AY221247), respectively; 67\% identity with Citrus unshiu CuFLS (AB011796); 
64\% identity with Gentiana triflora GtFLS (AB587658); and 63\% identity with Camellia nitidissima CnFLS (ADZ28516). Moreover, MaFLS harbored characteristic conserved ferrous iron-binding residues (His216, Asp218, and His272; marked by black arrows; Figure 1) and the 2-oxoglutarate binding residues (Arg282 and Ser284; marked by gray arrows; Figure 1) of FLS proteins. This clearly indicated that MaFLS belonged to the soluble $\mathrm{Fe}^{2+} / 2$-ODD protein family. Furthermore, MaFLS possessed five key amino acids (Tyr127, Phe129, Lys197, Phe288, and Ser290; marked by black dots; Figure 1) that were identified as potential active DHQ binding site residues, highly conserved in FLSs of various species [26]. In addition, MaFLS contained the FLS-specific motifs "PxxxIRxxxEQP" and "SxxTxLVP", which can be used to distinguish FLSs from other plant 2-ODDs, like F3H, ANS, and FNS. MaFLS possessed the residues responsible for the proper folding of the FLS polypeptide (Gly65 and Gly256; marked by asterisks; Figure 1), which are conserved in all 2-ODD proteins [17].

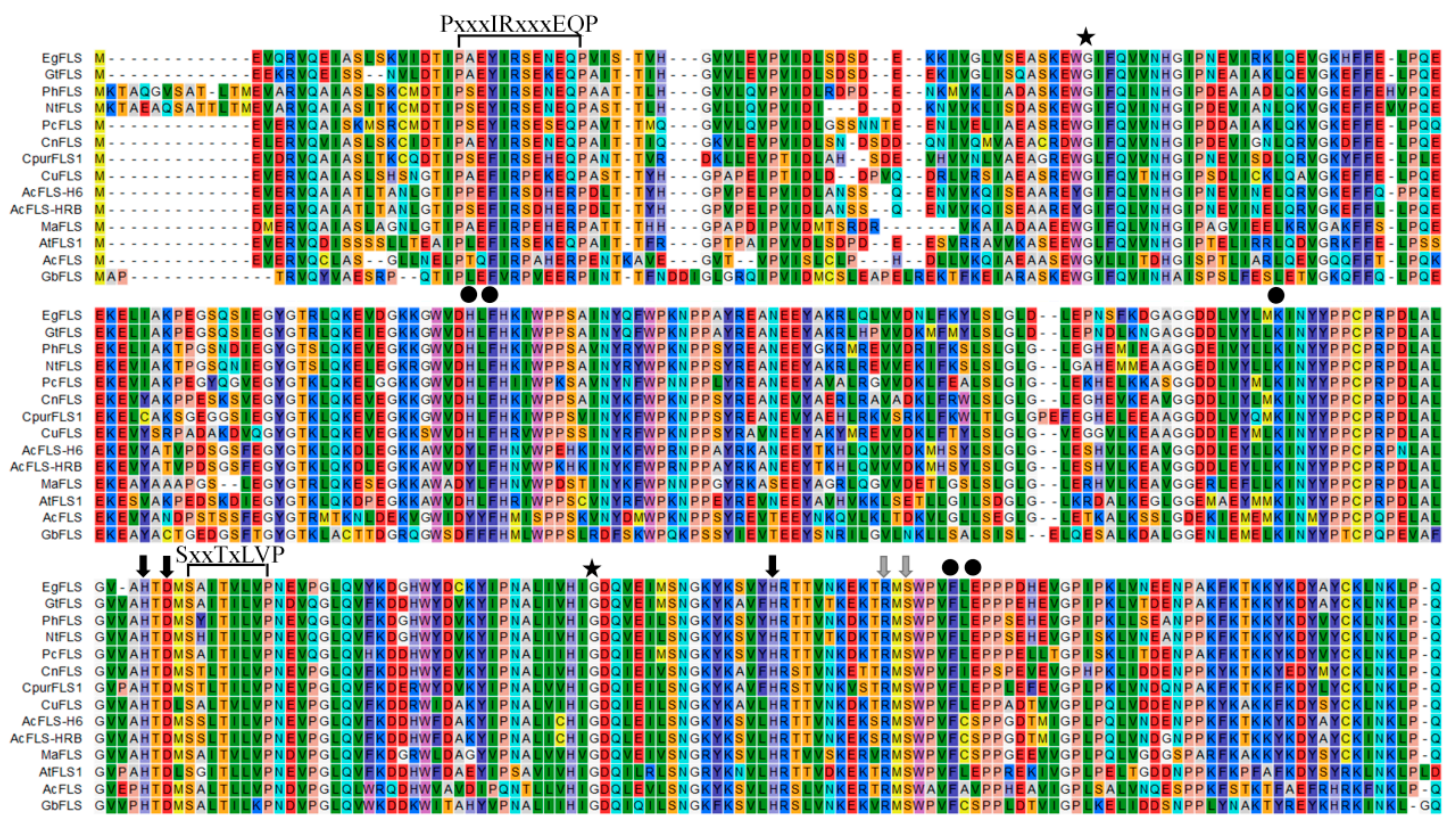

Figure 1. Multiple alignment of the predicted amino acid sequence of MaFLS with that of other flavonol synthase (FLS) proteins. MaFLS is compared with FLS sequences from Eustoma grandiflorum (EgFLS), Gentiana triflora (GtFLS), Petunia hybrida (PhFLS), Nicotiana tabacum (NtFLS), Petroselinum crispum (PcFLS), Camellia nitidissima (CnFLS), Cyclamen purpurascens (CpurFLS1), Citrus unshiu (CuFLS), Allium cepa (AcFLS-H6 and AcFLS-HRB), Arabidopsis thaliana (AtFLS1), Acacia confusa (AcFLS), and Ginkgo biloba (GbFLS). The alignment was generated using CLC sequence viewer 8.0. The FLS-specific motifs "PxxxIRxxxEQP" and "SxxTxLVP" are indicated. Black arrows indicate the ferrous iron-binding residues (His216, Asp218, and His272). Gray arrows represent the 2-oxoglutarate binding residues (Arg282 and Ser284). Black dots show the putative DHQ-binding residues (Tyr127, Phe129, Lys197, Phe288, and Ser290). Asterisks indicate the residues responsible for the proper folding of the FLS polypeptide (Gly65 and Gly256).

To determine the relationship between the putative MaFLS protein and other plant FLSs, we performed a phylogenetic analysis with the functionally characterized plant FLSs as well as various putative FLSs (Figure 2). MaFLS was grouped with fellow monocots close to Narcissus tazetta NtaFLS (AFS63900), Allium cepa AcFLS-H6 (AY221247), and AcFLS-HRB (AY647262) in the order Asparagales, and Lilium regale LrFLS (ASV46329) in the order Liliales (Figure 2). MaFLS showed a marked separation from enzymes of dicot plants and of monocot Poaceae grasses. These results indicate that the phylogenetic analysis fits well with the genetic relationships among the species. 


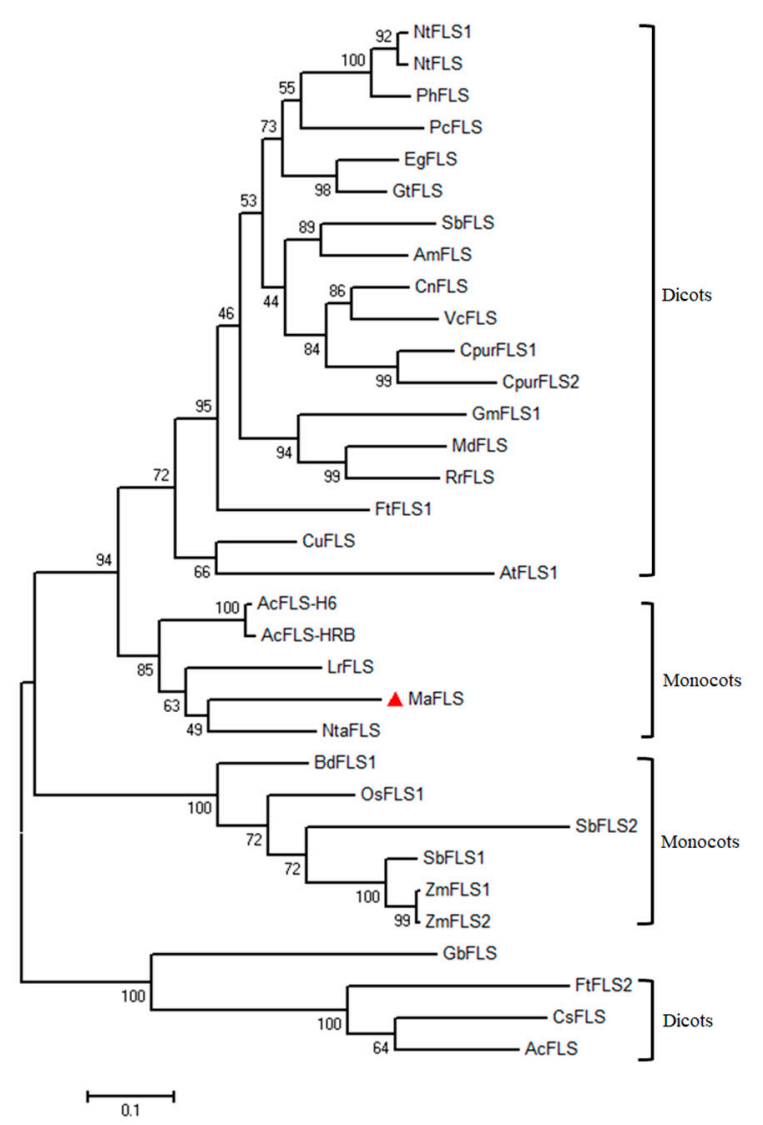

Figure 2. Phylogenetic analysis of MaFLS and other FLS proteins. The maximum-likelihood phylogenetic tree was generated using MEGA 6.0 software. Numbers at each interior branch indicate the bootstrap values of 1000 replicates. The bar indicates a genetic distance of 0.1. GenBank accession numbers are as follows: Muscari aucheri MaFLS (MH636605, marked with red triangle), Nicotiana tabacum NtFLS1 (ABE28017) and NtFLS (AB289451), Petunia hybrida PhFLS (CAA80264), Petroselinum crispum PcFLS (AAP57395), Eustoma grandiflorum EgFLS (AAF64168), Gentiana triflora GtFLS (AB587658), Scutellaria baicalensis SbFLS (KC404852), Antirrhinum majus AmFLS (ABB53382), Camellia nitidissima CnFLS (ADZ28516), Vaccinium corymbosum VcFLS (KP334105), Cyclamen purpurascens CpurFLS1(LC210072) and CpurFLS2 (LC210073), Glycine max GmFLS1 (AB246668), Malus domestica MdFLS (AY965343), Rosa rugosa RrFLS (KM099095), Fagopyrum tataricum FtFLS1 (JF274262) and FtFLS2 (JX401285), Citrus unshiu CuFLS (AB011796), Arabidopsis thaliana AtFLS1 (AAB41504), Allium cepa AcFLS-H6 (AY221247) and AcFLS-HRB (AY647262), Lilium regale LrFLS(ASV46329), Narcissus tazetta NtaFLS (AFS63900), Brachypodium distachyon BdFLS1 (XM_003570514), Oryza sativa OsFLS1 (NP_001048230), Sorghum bicolor SbFLS1 (XP_002454608) and SbFLS2 (EES00073), Zea mays ZmFLS1(BT039956) and ZmFLS2 (XP_008646309), Ginkgo biloba GbFLS (ACY00393), Camellia sinensis CsFLS (ABM88786), and Acacia confusa AcFLS (JN812062).

\subsection{Correlation Analysis of MaFLS Expression Levels and Total Flavonol Content}

We extracted metabolites from roots, bulbs, leaves, and petals at the five floral developmental stages (S1-S5) of "White Beauty" and "Dark Eyes" (Figure 3A). HPLC analysis revealed that total flavonol content in all organs of "White Beauty" was significantly higher than that of "Dark Eyes". In "White Beauty", total flavonol content increased during flower development, peaked before blooming, and then decreased gradually after petal expansion (Figure 3B). However, in "Dark Eyes", the total flavonol content was higher in S2 buds than in the buds at other developmental stages. In addition, total flavonols also accumulated in other vegetative organs, especially in leaves of the two grape hyacinth cultivars. Recently, Lou et al. reported that the level of total anthocyanins in violet-blue flowers such as those of "Dark Eyes" was substantially higher than that in the white flowers of 
"White Beauty" [22]. Therefore, these results likely reflect relatively higher flavonol accumulation and extremely low anthocyanin accumulation during the formation of white flowers in "White Beauty".

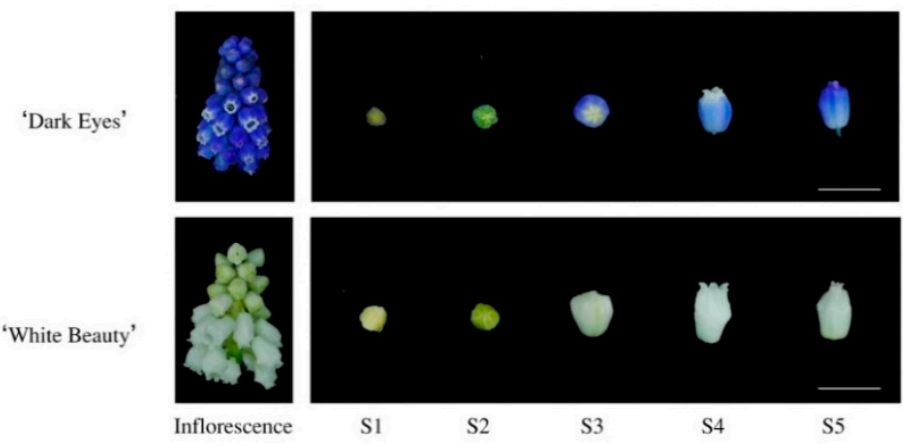

(A)

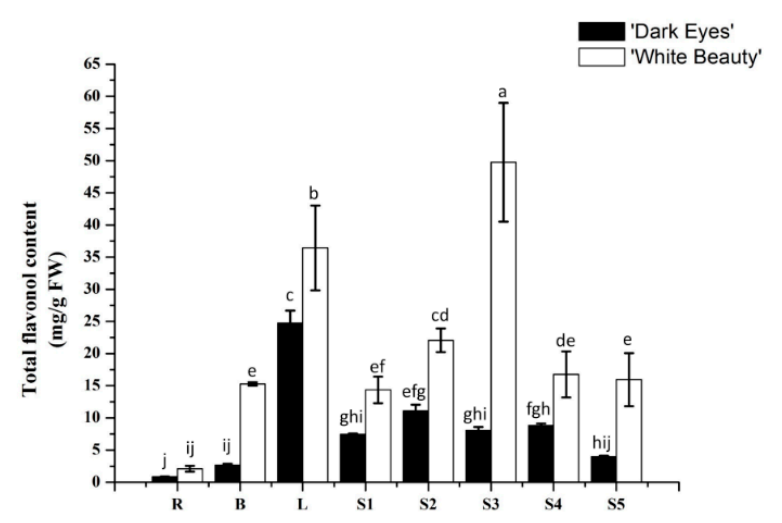

(B)

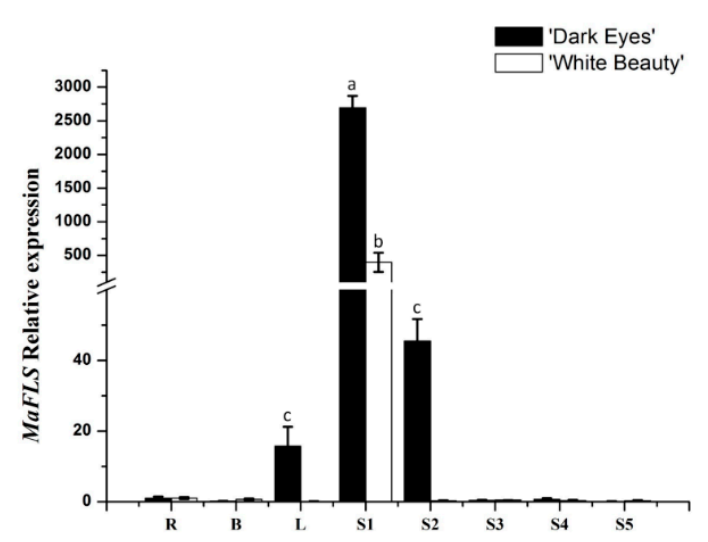

(C)

Figure 3. The flavonol content and expression profiles of MaFLS in M. aucheri "Dark eyes" and M. aucheri "White Beauty". (A) The inflorescence and the petals at five flower developmental stages of $M$. aucheri "Dark eyes" and M. aucheri "White Beauty". Bars, $5 \mathrm{~mm}$. (B) The total flavonol content of petals. FW: Fresh weight. (C) The expression profile of MaFLS. Flavonol content and MaFLS expression were determined in roots (R), bulbs (B), leaves (L), and petals at five flower developmental stages (S1-S5). MaActin was used as the internal expression control. Each column represents means \pm SD from three technical replicates. Different letters above the bars indicate significantly different values $(P<0.05)$ calculated using one-way ANOVA followed by a Duncan's multiple range test.

Furthermore, we determined the expression levels of MaFLS in different organs and flower development stages by qRT-PCR analysis. MaFLS transcript levels were highest in S1 buds, whereas 
MaFLS expression was undetectable in S2-5 petals and in the roots, bulbs, and leaves of M. aucheri "White Beauty" (Figure 3C). Similarly, in M. aucheri "Dark eyes", MaFLS expression was extraordinarily high in S1 buds, markedly lower in S2 petals, and undetectable in S3-5 petals. Moreover, MaFLS expression was barely detectable in the roots and bulbs, and slightly detectable in the leaves of M. aucheri "Dark eyes" (Figure 3C). Thus, MaFLS is predominantly expressed in the early stages of flower development in grape hyacinth, which is consistent with reports in other ornamental plants, such as carnation (Dianthus caryophyllus), petunia, lisianthus, and cyclamen $[1,6,18,28]$. Together, our results show that MaFLS likely plays a key role in flower coloration, alongside other copigmentation-related FLS genes. Nevertheless, MaFLS expression is not concomitant with the accumulation of total flavonols in grape hyacinth.

\subsection{In Vivo Localization of MaFLS}

To detect the subcellular localization of MaFLS, the positive control 35S:GFP (pBI221) and the recombinant plasmid pBI221-MaFLS-GFP were transformed into $A$. thaliana mesophyll protoplasts. As shown in Figure 4, fluorescence from 35S:GFP was dispersed throughout $A$. thaliana mesophyll protoplasts, whereas fluorescence from the MaFLS:GFP fusion protein was mainly localized in the cytosol and cell periphery. Therefore, MaFLS is both a cytoplasmic and cell periphery protein.

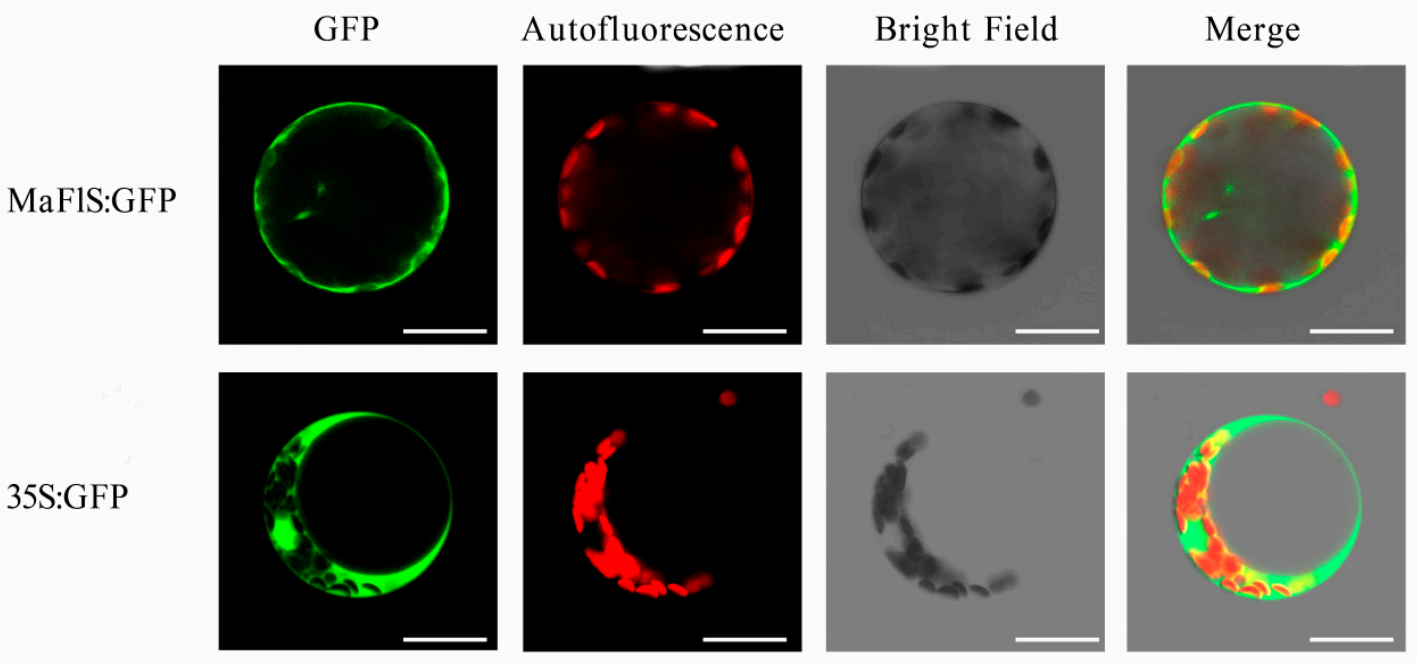

Figure 4. Subcellular localization of MaFLS proteins. Transient expression of the MaFLS-GFP fusion protein and the 35S:GFP control in A. thaliana mesophyll protoplasts. GFP, GFP fluorescence; autofluorescence, chloroplast autofluorescence; merge is an overlay of chloroplast autofluorescence, GFP fluorescence, and bright-field images. Bars, $25 \mathrm{~mm}$.

\subsection{Heterologous Expression of MaFLS in Tobacco Alters Petal Color}

To ascertain the function of MaFLS in flower coloration, MaFLS was transformed into tobacco ( $N$. tabacum "NC89") under the control of the 35S-CaMV promoter for heterologous expression experiments. We identified 11 transformant lines by PCR analysis, using the vector-specific primers 2300-F and 2300-R (Table S1). Compared with the deep pink flowers of wild-type tobacco, the transgenic tobacco lines heterologously expressing MaFLS showed reduced levels of petal pigmentation (Figure 5A). Five lines displayed a severe phenotype $(S)$ of pale pink to completely white petals; three lines displayed a medium phenotype $(\mathrm{M})$ of pink petals, whereas the remaining lines displayed a weak phenotype (W) of pale pink petals (Figure 5A). 

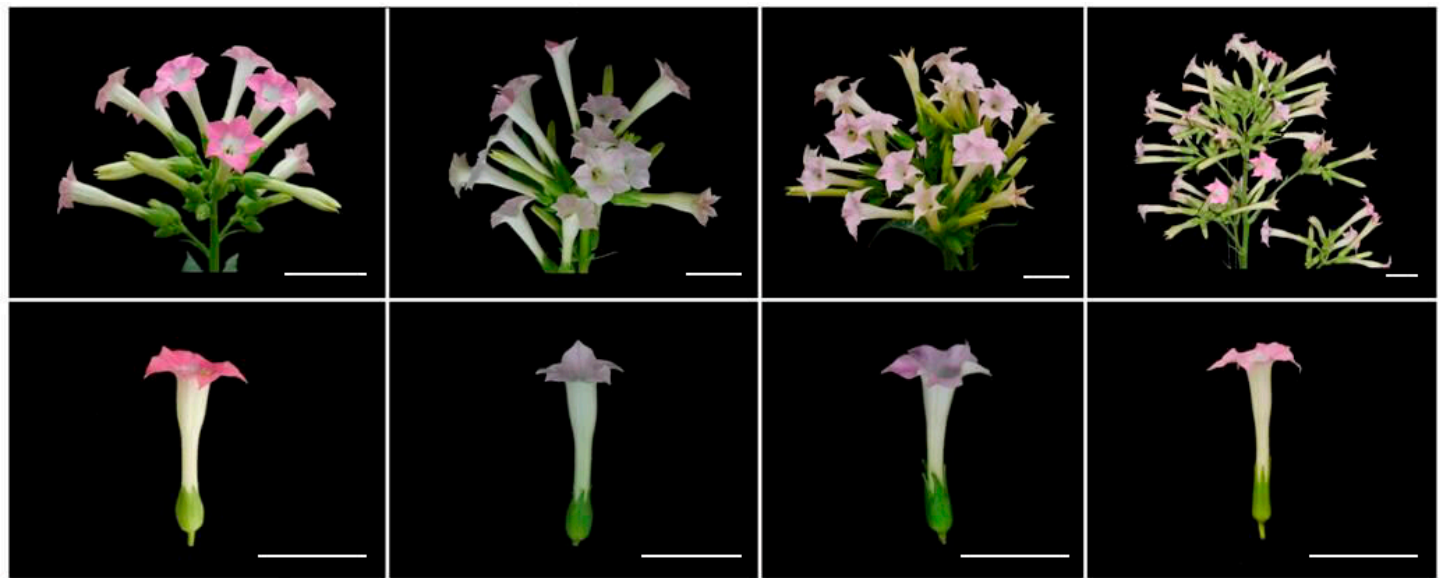

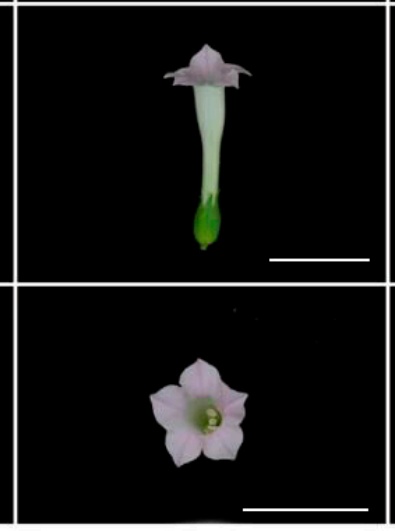

$\mathrm{S}$

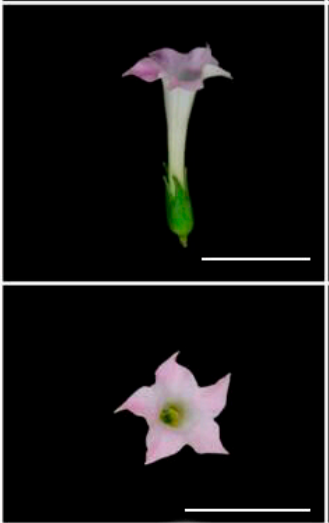

M

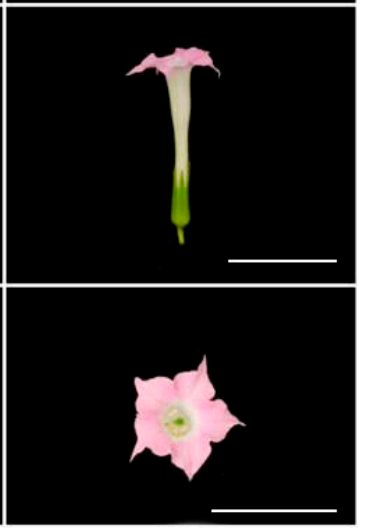

W

(A)

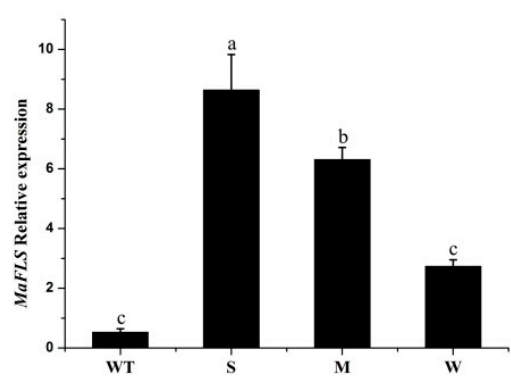

(B)

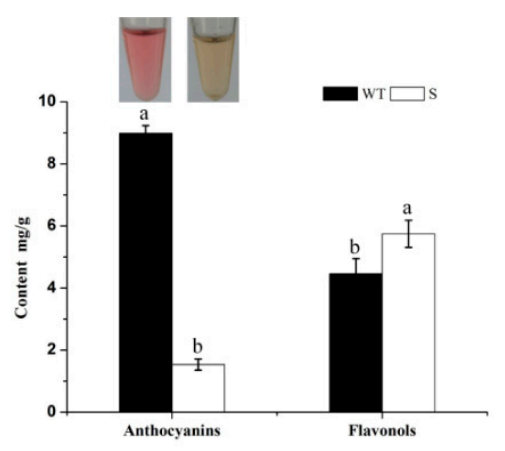

(C)

Figure 5. Changes in tobacco flowers induced by heterologous expression of MaFLS. (A) Heterologous expression of MaFLS in transgenic tobacco flowers resulted in a clear phenotypic change in petal coloration. Shown are non-transformed controls (WT) and three MaFLS-expressing lines exhibiting different phenotypic characteristics ( $\mathrm{S}$, strong; $\mathrm{M}$, medium; W, weak). Bars, $5 \mathrm{~cm}$. (B) qRT-PCR analysis of MaFLS relative expression levels in the petals of three independent transgenic tobacco lines. NtTubA1 was used as the internal expression control. (C) The color of the extractions is shown in the centrifugal tubes. HPLC analysis of anthocyanin and flavonol levels in tobacco petals in $\mathrm{mg} / \mathrm{g}$ fresh weight (FW) of the non-transformed control (wild type, WT) and the transgenic line with a strong phenotype (S). Each column represents means \pm SD from three technical replicates. Different letters above the bars indicate significantly different values $(P<0.05)$ calculated using one-way ANOVA followed by Duncan's multiple range test.

To further investigate MaFLS expression levels in the transgenic tobacco lines, we carried out qRT-PCR analysis using MaFLS-specific qRT-PCR primers (Table S1). We discovered that the extent of change in petal coloration was consistent with the relative expression levels of MaFLS in the transgenic tobacco lines, in that the lines with a severe and a weak phenotype had the highest and lowest MaFLS expression levels, respectively (Figure 5B). Therefore, the changes in petal color in the transgenic tobacco lines appear to be the result of heterologous MaFLS expression. 
In addition, we further investigated the changes in anthocyanin and flavonol levels in petals from transgenic tobacco lines with a severe phenotype by HPLC. The color of the extractions indicated that the petals of lines with a severe phenotype contained less anthocyanins than the non-transformed control (Figure 5C). This was confirmed by HPLC analysis, which showed that the severe phenotype lines had a clear reduction in total anthocyanin levels; however, they accumulated higher levels of total flavonols, compared to the non-transformed control (Figure 5C). These results strongly suggest that high-level MaFLS expression in transgenic tobacco lines causes a remarkable decrease in anthocyanin content and an increase in flavonol accumulation in vivo, which results in a severe reduction in petal pigmentation.

\subsection{Heterologous Expression of MaFLS in Tobacco Affects the Expression of Anthocyanin Pathway Genes}

Subsequently, we conducted a qRT-PCR expression analysis of endogenous genes involved in the flavonoid biosynthetic pathway in the petals of MaFLS-expressing transgenic tobacco (Figure 6A, primers are listed in Table S1). The analyzed genes included twelve structural genes (NtPAL, NtC4H, $N t 4 C L, N t C H S, N t C H I, N t F 3 H, N t F 3^{\prime} H, N t F 3^{\prime} 5^{\prime} H, N t F L S, N t D F R, N t A N S$, and NtUFGT) and three regulatory genes (NtAN2, NtAN1a, and NtAN1b), which are highlighted in red (Figure 6A).

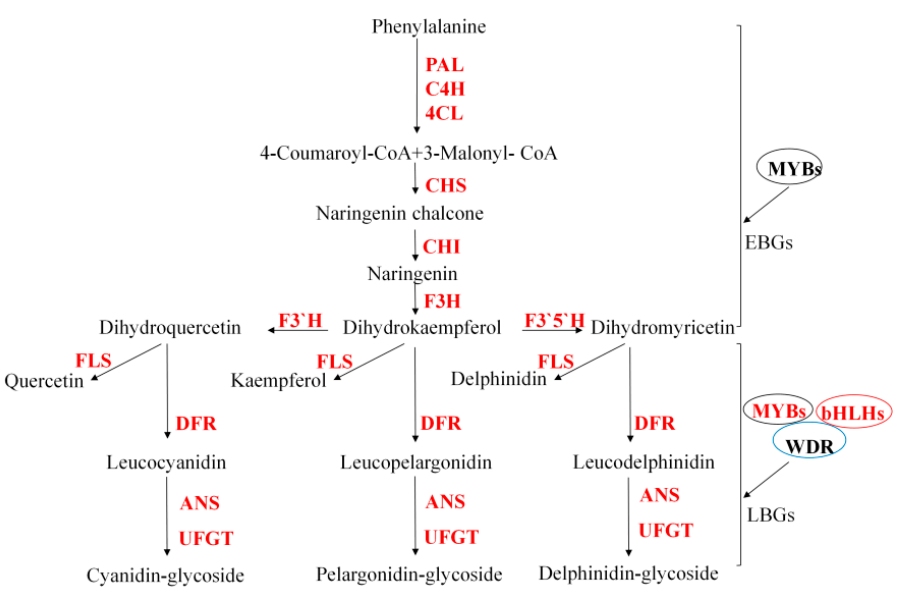

(A)

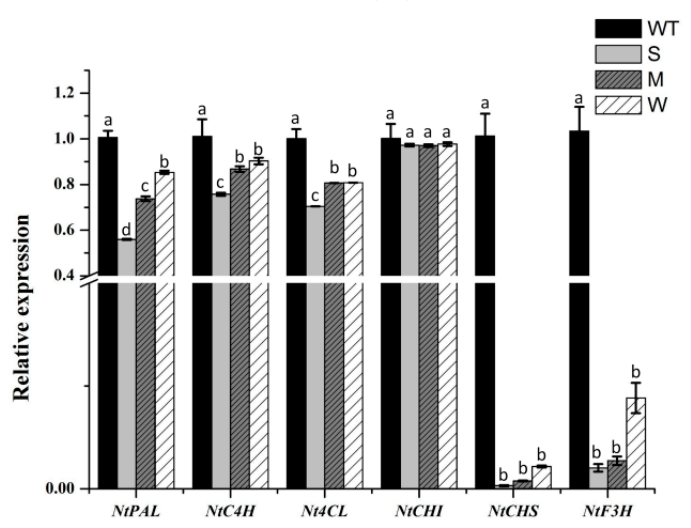

(B)

Figure 6. Cont. 


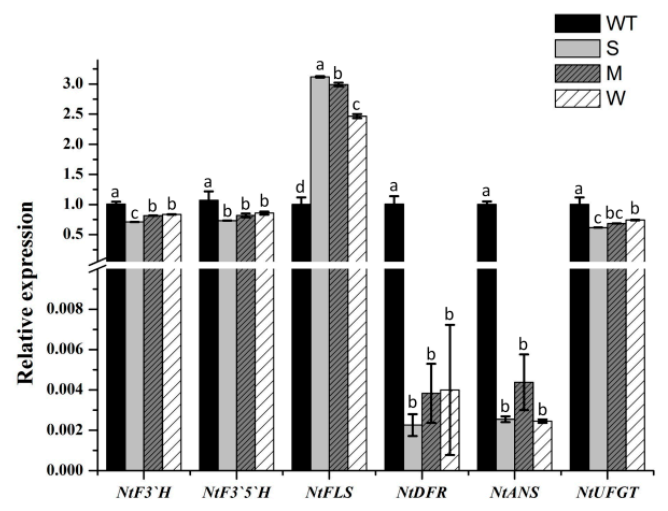

(C)

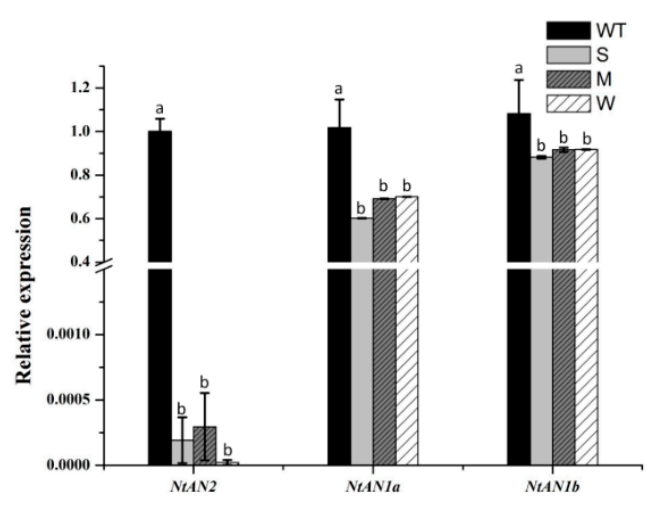

(D)

Figure 6. A schematic diagram of flavonoid biosynthetic pathway and expression profiles of anthocyanin biosynthetic endogenous TF genes in petals. (A) A schematic diagram of flavonoid biosynthetic pathway. EBGs, the early biosynthetic genes; LBGs, the late biosynthetic genes; PAL, phenylalanine ammonia-lyase; $\mathrm{C} 4 \mathrm{H}$, cinnamate 4-hydroxylase; 4CL, 4-coumarate: CoA ligase; $\mathrm{CHS}$, chalcone synthase; $\mathrm{CHI}$, chalcone isomerase; $\mathrm{F} 3 \mathrm{H}$, flavanone 3-hydroxylase; $\mathrm{F3}$ ' $\mathrm{H}$, flavonoid 3'-hydroxylase; F3'5'H, flavonoid 3'5'-hydroxylase; FLS, flavonol synthase; DFR, dihydroflavonol 4-reductase; ANS, anthocyanidin synthase; UFGT, uridine diphosphate-sugar: Flavonoid glycosyltransferases; MYBs, MYB transcription factors; bHLHs, basic helix-loop-helix proteins; WDR, WD repeat protein. The analyzed genes are highlighted in red. (B)The expression patterns of $N t P A L, N t C 4 H, N t 4 C L, N t C H S$, $N t C H I$, and $F 3 H$ in petals. (C) The expression patterns of $N t F 3^{\prime} H, N t F 3^{\prime} 5^{\prime} H, N t F L S, N t D F R$, NtANS, and NtUFGT in petals. (D) The expression patterns of three regulatory genes (NtAN2, NtAN1a, and NtAN1b) in petals. Data are for the non-transformed control (WT) and three MaFLS-expressing lines exhibiting different phenotypic characteristics ( $\mathrm{S}$, strong; $\mathrm{M}$, medium; W, weak). Each column represents means \pm $\mathrm{SD}$ from three technical replicates. Different letters above the bars indicate significantly different values $(P<0.05)$ calculated using one-way ANOVA followed by Duncan's multiple range test.

Based on the one-way ANOVA statistical results presented in Figure 6, only the expression level of $\mathrm{NtCHI}$ was not significantly different between the MaFLS-expressing lines and the non-transformed control; all the other genes showed the decreased expressions. In particular, NtCHS, NtF3H, NtDFR, $N t A N S$, and NtAN2 expression was remarkably lower in the MaFLS-expressing lines than in the non-transformed control (Figure 6B-D). Particularly, the transcript levels of two endogenous genes (NtDFR and NtANS) and one regulatory gene (NtAN2), closely related to anthocyanin biosynthesis, showed remarkable reductions in three MaFLS-expressing lines, compared with the non-transformed control (Figure 6C,D). Conversely, the expression of endogenous NtFLS was markedly upregulated in the MaFLS-expressing lines. These results suggest that heterologous expression of MaFLS in tobacco petals substantially inhibited expression of the key structural genes (NtDFR and NtANS) and the vital 
regulatory gene (NtAN2) that have been implicated in anthocyanin biosynthesis and upregulated the expression of endogenous NtFLS.

\section{Discussion}

Anthocyanins and flavonols are important flavonoid phytochemicals that contribute to flower coloration. Understanding the molecular and biochemical controls of anthocyanin and flavonol pathways, or studying related genes, is an important focus in the ornamental plant industry, since novel color morphs can be profitable. Here, we describe the cloning and molecular characterization of MaFLS, the first FLS characterized from ornamental monocots. Sequence alignments revealed that MaFLS possesses the HxDxnH and RxS motifs for binding ferrous iron and 2-OG, respectively. Moreover, MaFLS has the five amino acid residues (Tyr127, Phe129, Lys197, Phe288, and Ser290; Figure 1) responsible for substrate binding [26]. It is noteworthy that Tyr127 and Ser290 are not conserved. Similar to Tyr132 in AcFLSs and Phe137 in GbFLS [14,17], the presence of Tyr127 in MaFLS (instead of the His, residue commonly present in other FLSs at this position) might impart improved enzyme catalytic activity for quercetin production [26]. Because the previous study showed that it is a potentially attractive substrate binding site for modification to engineer a FLS with improved activity [26].

Some progress has recently been made in analyzing the anthocyanin profiles of Muscari [22,24,29-31]. However, few reports have focused on the flavonol profiles in Muscari. Our previous work revealed that flavonols in grape hyacinth petals are mainly derivatives of kaempferol and quercetin, in accordance with flavonol accumulation in lisianthus petals [32]. Here, we determined the levels of total flavonols in two grape hyacinth cultivars, one with white flowers and the other with blue. We found that total flavonol content of the white-flower cultivar "White Beauty" was significantly higher than that of blue-flower cultivar "Dark Eyes". How flavonols regulate flower color in these cultivars is unclear, but one possibility is that they act as copigments that participate in petal coloration.

In addition, we observed little correlation between the expression levels of MaFLS and the accumulation of total flavonols in grape hyacinth. Conversely, positive correlations between flavonoid concentration and mRNA levels of FLS genes have been reported in other plant species [19]. First, this correlation depends on the availability of primary substrate (dihydroflavonols) flux in the pathway, because only some of them can be catalyzed by MaFLS to the corresponding flavonols, while others may synthesize many other molecules that were not measured in this analysis. Second, this phenomenon perhaps indicates that other MaFLS isogenes may be expressed to produce the appropriate flavonols in grape hyacinth, as is the case in A. thaliana, lisianthus, and onion $[1,9,17]$. However, only one putative MaFLS sequence has been identified thus far. Due to a lack of sufficient genomic resources, we are unaware of other FLS genes that exist in grape hyacinth. Nevertheless, we speculate that the MaFLS characterized here promotes flavonol production to act as UV-B sunscreens and protect young developing floral tissue and that these colorless flavonols may act as copigments sandwiched between anthocyanin molecules to induce a blue shift [1,12]. Furthermore, these colorless flavonols might act as UV-spectrum flower pigments, contributing to the attractive and defensive functions for insects [33], or they might be important for male fertility and auxin metabolism and transport [34,35].

At the subcellular level, MaFLS appears to localize to the cytoplasm and cell periphery, but not to the nucleus, in accordance with the observation that flavonoids are synthesized and localized to the cytoplasm in Arabidopsis [36]. However, there are many flavonoid metabolic enzymes for which dual cytoplasmic/nuclear localization has been observed, such as CHS, CHI, and FLS [35].

We have shown that constitutive heterologous expression of MaFLS influenced flower color in transgenic tobacco lines. HPLC analysis showed a dramatic decrease in the levels of total anthocyanins and an increase in the levels of total flavonols in the resulting tobacco flowers. The severity of the flower color phenotype was highly consistent with the MaFLS transgene expression level. It is noteworthy that over-expression of MaFLS in tobacco inhibited expression of the genes involved in the early step of flavonoid biosynthesis, particularly dampening the expression of the key structural genes 
(NtDFR and NtANS) and the vital regulatory gene (NtAN2) in the anthocyanin synthesis pathway. This phenomenon may be explained as a feedback mechanism existing in the flavonoid pathway that affected the expression of genes in the early step of flavonoid biosynthesis [20]. The result also further demonstrated that the competition between FLS and DFR genes ultimately determines the flower coloration.

\section{Materials and Methods}

\subsection{Plant Materials and Growth Conditions}

Grape hyacinth (M. aucheri "White Beauty" and M. aucheri "Dark Eyes") plants were grown in the experimental field of the Northwest A\&F University at Yangling Distruct in Shaanxi Province, China. The petals of grape hyacinth were divided into five floral development stages (S1-S5), according to the previous description (Figure 3A) [22]. To obtain RNA and metabolite samples, healthy tissues and flowers were collected, frozen immediately in liquid nitrogen, and stored at $-80^{\circ} \mathrm{C}$. Tobacco plants (Nicotiana tabacum "NC89") for transformation were grown aseptically from seed on Murashige and Skoog medium, supplemented with 3\% (w/v) sucrose, and transgenic tobacco flowers were harvested at the full-bloom stage.

\subsection{Cloning of Full-Length MaFLS cDNAs}

A FLS homolog was identified in the transcriptome of grape hyacinth [24], designated MaFLS. To obtain the full-length cDNA sequence of MaFLS, we performed both $5^{\prime}$ - and $3^{\prime}$-RACE experiments, using the flowers of M. aucheri "White Beauty", as in the previous method [23]. The primers used for 501 and 3'-RACE PCR and full-length gene cloning are listed in Table S1. The obtained MaFLS cDNA sequence was submitted to the NCBI GenBank database (accession number MH636605).

\subsection{Sequence Alignment and Phylogenetic Analysis}

Full-length amino acid sequences of MaFLS and other FLS proteins were retrieved from the GenBank database. Multiple sequence alignments were performed with CLC sequence viewer 8.0 software. FLS-specific motifs and conserved amino acid residues were indicated by different colored symbols. The phylogenetic tree was constructed by the maximum likelihood method with 1000 bootstrap replicates using MEGA 6.0.

\subsection{RNA Isolation and qRT-PCR Analysis}

Total RNA was extracted from frozen tissue of the flowers, roots, bulbs, and leaves of grape hyacinth (M. aucheri "White Beauty" and M. aucheri "Dark eyes") as well as flowers of tobacco (NC89), using the Omega Total RNA Kit (Omega, Norcross, GA, USA). Purified RNA was assessed using agarose electrophoresis and measured on a Nanodrop 2000 (Thermo Scientific). Then, 1- $\mu$ g RNA aliquots were used for reverse transcription to cDNA, using the PrimeScript ${ }^{\mathrm{TM}}$ RT Reagent Kit (TaKaRa Biotechnology, Dalian, China). The cDNA was diluted five-fold and used as the template for qRT-PCR. NovoStart ${ }^{\circledR}$ SYBR qPCR SuperMix Plus (Novoprotein, Shanghai, China) was used as the fluorochrome for the qRT-PCR assay. The assay was conducted using the iQ5 RT-PCR detection system (Bio-Rad, Hercules, CA, USA). Each reaction mixture consisted of NovoStart ${ }^{\circledR}$ SYBR qPCR SuperMix Plus with $0.8 \mu \mathrm{L}$ of forward and reverse primers each, $1 \mu \mathrm{L}$ of cDNA, and $7.4 \mu \mathrm{L}$ of $\mathrm{ddH}_{2} \mathrm{O}$ in a final volume of $20 \mu \mathrm{L}$. The amplification protocol was $95^{\circ} \mathrm{C}$ for $1 \mathrm{~min}$, followed by 40 cycles of $95^{\circ} \mathrm{C}$ for $20 \mathrm{~s}, 58$ to $62{ }^{\circ} \mathrm{C}$ for $20 \mathrm{~s}$, and $72{ }^{\circ} \mathrm{C}$ for $30 \mathrm{~s}$. The qRT-PCR primers of grape hyacinth and tobacco are listed in Table S1. MaActin and NtTubA1 were used as the internal control genes in each grape hyacinth and tobacco sample, respectively. All analyses were conducted in technical triplicate. 


\subsection{Subcellular Localization of MaFLS}

To investigate the subcellular localization of MaFLS, the ORF of MaFLS without a termination codon was inserted between the $\mathrm{XbaI}$ and $\mathrm{KpnI}$ sites of the pBI221-GFP vector, using the Seamless Cloning and Assembly Kit (Novoprotein, China; primers are listed in Table S1) to generate the recombinant plasmid pBI221-MaFLS-GFP. Using the PEG-calcium mediated transfection method [37], the recombinant plasmid pBI221-MaFLS-GFP was transformed into A. thaliana mesophyll protoplasts. After 16-18 h of cultivation, the transformed protoplasts were observed with a confocal laser scanning microscope (TCS SP8, Leica, Wetzlar, Germany).

\subsection{Heterologous Expression Vector Construction and Stable Tobacco Transformation}

For constructing the MaFLS heterologous expression vector, the MaFLS ORF sequence without

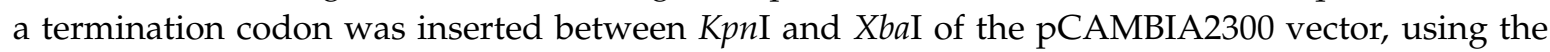
Seamless Cloning and Assembly Kit (Novoprotein, China; primers are listed in Table S1) to produce the recombinant plasmid p2300-MaFLS. Then, the recombinant plasmid p2300-MaFLS was introduced into Agrobacterium tumefaciens Gv3101 (MP) by electroporation. Tobacco leaf disk transformation was conducted using a previously described protocol [38]. T0 generation of transgenic tobacco lines heterologously expressing MaFLS were used for qPCR analysis, and the lines showing a severe phenotypic change in petal pigmentation were used for further HPLC analysis.

\subsection{HPLC Analysis}

Petals from the five floral developmental stages; roots, bulbs, and leaves of grape hyacinth; and the fresh petals of transgenic tobacco were ground to powder in liquid nitrogen and extracted with methanol to $\mathrm{H}_{2} \mathrm{O}$ to formic acid to trifluoroacetic acid $(70: 27: 2: 1, v / v / v / v)$ for analysis of total anthocyanins $[39,40]$ or extracted with methanol for analysis of total flavonols [41]. The supernatant was filtered through $0.22-\mu \mathrm{m}$ Millipore filters. Total anthocyanin content of tobacco flowers was measured using a UV-visible spectrophotometer (UV2600, Shimadzu, Kyoto, Japan) and calculated according to the equation: QAnthocyanins $=(\mathrm{A} 530-0.25 \times \mathrm{A} 657) \times \mathrm{M}^{-1}$.

Flavonol was detected at $360 \mathrm{~nm}$ using reverse HPLC analysis, as in the previous descriptions [22,23]. The results were expressed as milligrams of anthocyanins or flavonols per gram of fresh weight $(\mathrm{mg} / \mathrm{g} \mathrm{FW})$. All samples were analyzed in three biological replicates.

\subsection{Statistical Analysis}

Analysis of variance (one-way ANOVA) was performed using the SAS program (version 8.0, SAS Institute, Cary, NC, USA). The statistical difference was compared by the Duncan's multiple range test $(P<0.05$ was considered significant).

\section{Conclusions}

In this study, we identified a FLS gene, MaFLS, which is predominantly expressed in the early stages of flower development in grape hyacinth. Heterologous expression of MaFLS in tobacco showed a reduction in pigmentation in the petals because of a remarkable decrease in anthocyanin content and an increase in flavonol accumulation. Moreover, our study demonstrates the vital role of MaFLS, to promote flavonol production in young developing floral tissue, and that these flavonols may play a key role in flower coloration in grape hyacinth. However, MaFLS-based regulation does not seem to fully explain the flower coloration of grape hyacinth. Future research should be conducted to determine the following: (1) How MaFLS and MaDFR gene products compete for common substrates to regulate flavonoid biosynthesis and coloration, (2) how flavonol and anthocyanin biosynthesis is regulated under the control of transcriptional regulators, and (3) how the precise ratio of flavonol and anthocyanin metabolites affects flower coloration. 
Supplementary Materials: The following are available online.

Author Contributions: L.D. and Y.L. conceived and designed the research. H.L., B.S., H.Z., J.G., and B.Z. conducted the experiments. H.L. analyzed the data. L.D. and H.L. contributed to the writing of the manuscript. All authors read and approved the manuscript.

Funding: The authors would like to thank the anonymous reviewers for their comments. This work was supported by the National Natural Science Foundation of China (Grant No. 31700625 and No. 31471905), Shaanxi Province Science and Technology Research and Development Program (2017JQ3019), and the Fundamental Research Funds for the Central Universities (Z109021603).

Conflicts of Interest: The authors declare that they have no conflict of interests.

$\begin{array}{ll}\text { Abbreviations } & \\ \text { FLS } & \text { flavonol synthase. } \\ \text { DFR } & \text { dihydroflavonol 4-reductase } \\ \text { ANS } & \text { anthocyanidin synthase } \\ \text { qRT-PCR } & \text { quantitative real-time PCR } \\ \text { DHK } & \text { dihydrokaempferol } \\ \text { DHQ } & \text { dihydroquercetin } \\ \text { ORF } & \text { open reading frame } \\ \text { PCR } & \text { polymerase chain reaction } \\ \text { UFGT } & \text { glucosyltransferase } \\ \text { 2-ODD } & \text { 2-oxoglutarate-dependent dioxygenase }\end{array}$

\section{References}

1. Nielsen, K.; Deroles, S.; Markham, K.; Bradley, M.; Podivinsky, E.; Manson, D. Antisense flavonol synthase alters copigmentation and flower color in lisianthus. Mol. Breed. 2002, 9, 217-229. [CrossRef]

2. Davies, K.; Schwinn, K.; Deroles, S.; Manson, D.; Lewis, D.; Bloor, S.; Bradley, J. Enhancing anthocyanin production by altering competition for substrate between flavonol synthase and dihydroflavonol 4-reductase. Euphytica 2003, 131, 259-268. [CrossRef]

3. Brouillard, R.; Dangles, O. Flavonoids and flower colour. In The Flavonoids, Advances in Research since 1986; Harborne, J.B., Ed.; Chapman \& Hall: London, UK, 1993; pp. 565-588.

4. Zhou, X.; Fan, Z.; Chen, Y.; Zhu, Y.; Li, J.; Yin, H. Functional analyses of a flavonol synthase-like gene from Camellia nitidissima reveal its roles in flavonoid metabolism during floral pigmentation. J. Bio. Sci. 2013, 38, 593-604. [CrossRef]

5. Britsch, L.; Heller, W.; Grisebach, H. Conversion of flavanone to flavone, dihydroflavonol and flavonol with an enzyme system from cell culture of parsley. Z. Naturforsch 1981, 36, 742-750. [CrossRef]

6. Holton, T.; Brugliera, F.; Tanaka, Y. Cloning and expression of flavonol synthase from Petunia hybrida. Plant J. 1993, 4, 1003-1010. [CrossRef]

7. Martens, S.; Preuß, A.; Matern, U. Multifunctional flavonoid dioxygenases: Flavonol and anthocyanin biosynthesisin Arabidopsis thaliana L. Phytochem. 2010, 71, 1040-1049. [CrossRef] [PubMed]

8. Pelletier, M.; Murrell, J.; Shirley, B. Characterization of flShirle synthase and leucoanthocyanidin dioxygenase genes in Arabidopsis-further evidence for differential regulation of early and late genes. Plant Physiol. 1997, 113, 1437-1445. [CrossRef]

9. Owens, D.; Alerding, A.; Crosby, K.; Bandara, A.; Westwood, J.; Winkel, B. Functional analysis of a predicted favonol synthase gene family in Arabidopsis. Plant Physiol. 2008, 147, 1046-1061. [CrossRef]

10. Preuß, A.; Stracke, R.; Weisshaar, B.; Hillebrecht, A.; Matern, U.; Martens, S. Arabidopsis thaliana expresses a second functional flavonol synthase. FEBS Lett. 2009, 583, 1981-1986. [CrossRef] [PubMed]

11. Moriguchi, T.; Kita, M.; Ogawa, K.; Tomono, Y.; Endo, T.; Omura, M. Flavonol synthase gene expression during citrus fruit development. Physiol. Plant 2002, 114, 251-258. [CrossRef] [PubMed]

12. Takahashi, R.; Githiri, S.; Hatayama, K.; Dubouzet, E.; Shimada, N.; Aoki, T.; Ayabe, S.; Iwashina, T.; Toda, K.; Matsumura, H. A single-base deletion in soybean flavonol synthase gene is associated with magenta flower color. Plant Mol. Biol. 2007, 63, 125-135. [CrossRef] 
13. Kimura, S.; Nakatsuka, T.; Yamada, E.; Saito, M.; Nishihara, M. A flavonol synthase gene GtFLS defines anther-specific flavonol accumulation in gentian. Plant Biotechnol. 2010, 28, 211-221. [CrossRef]

14. Xu, F.; Li, L.; Zhang, W.; Cheng, H.; Sun, N.; Cheng, S.; Wang, Y. Isolation, characterization, and function analysis of a flavonol synthase gene from Ginkgo biloba. Mol. Biol. Rep. 2012, 39, 2285-2296. [CrossRef]

15. Ferreyra, F.; Rius, S.; Emiliani, J.; Pourcel, L.; Feller, A.; Morohashi, K.; Casati, P.; Grotewold, E. Cloning and characterization of a UV-B inducible maize fl E. Cl synthase. Plant J. 2010, 62, 77-91. [CrossRef]

16. Zhang, C.; Liu, H.; Jia, C.; Liu, Y.; Wang, F.; Wang, J. Cloning, characterization and functional analysis of a flavonol synthase from Vaccinium corymbosum. Trees 2016, 30, 1595-1605. [CrossRef]

17. Park, S.; Kim, D.; Lee, J.; Ha, S.; Lim, S. Comparative analysis of two flavonol synthases from different-colored onions provides insight into flavonoid biosynthesis. J. Agric. Food Chem. 2017, 65, 5287-5298. [CrossRef]

18. Akita, Y.; Kitamura, S.; Mikami, R.; Ishizaka, H. Identification of functional flavonol synthase genes from fragrant wild cyclamen (Cyclamen purpurascens). J. Plant Biochem. Biotechnol. 2018, 27, 147-155. [CrossRef]

19. Liu, W.; Xiao, Z.; Fan, C.; Jiang, N.; Meng, X.; Xiang, X. Cloning and characterization of a flavonol synthase gene from Litchi chinensis and its variation among litchi cultivars with different fruit maturation periods. Front. Plant Sci. 2018, 9, 567. [CrossRef]

20. Luo, P.; Ning, G.; Wang, Z.; Shen, Y.; Jin, H.; Li, P.; Huang, S.; Zhao, J.; Bao, M. Disequilibrium of flavonol synthase and dihydroflavonol-4-reductase expression associated tightly to white vs. red color flower formation in plants. Front. Plant. Sci. 2016, 6, 1257. [CrossRef]

21. Yuan, Y.; Rebocho, A.; Sagawa, J.; Stanley, L.; Bradshaw, H. Competition between anthocyanin and flavonol biosynthesis produces spatial pattern variation of floral pigments between Mimulus species. PNAS 2016, 113, 2448-2453. [CrossRef]

22. Lou, Q.; Wang, L.; Liu, H.; Liu, Y. Anthocyanin profiles in flowers of grape hyacinth. Molecules 2017, $22,688$. [CrossRef] [PubMed]

23. Chen, K.; Liu, H.; Lou, Q.; Liu, Y. Ectopic expression of the grape hyacinth (Muscari armeniacum) R2R3-MYB transcription factor gene, MaAN2, induces anthocyanin accumulation in tobacco. Front. Plant Sci. 2017, 8, 965. [CrossRef]

24. Lou, Q.; Liu, Y.; Qi, Y.; Jiao, S.; Tian, F.; Jiang, L.; Wang, Y. Transcriptome sequencing and metabolite analysis reveals the role of delphinidin metabolism in flower colour in grape hyacinth. J. Exp. Bot. 2014, 65, 3157-3164. [CrossRef]

25. Jiao, S.; Liu, Y.; Lou, Q.; Jiang, L. Cloning and expression analysis of dihydroflavonol 4-reductase gene (DFR) from grape hyacinth (Muscari armeniacum). J. Agric. Biotechnol. 2014, 22, 529-540. (in Chinese).

26. Chua, C.; Biermann, D.; Goo, K.; Sim, T. Elucidation of active site residues of Arabidopsis thaliana flavonol synthase provides a molecular platform for engineering flavonols. Phytochemistry 2008, 69, 66-75. [CrossRef]

27. Stracke, R.; De Vos, R.; Bartelniewoehner, L.; Ishihara, H.; Sagasser, M.; Martens, S.; Weisshaar, B. Metabolomic and genetic analyses of flavonol synthesis in Arabidopsis thaliana support the in vivo involvement of leucoanthocyanidin dioxygenase. Planta 2009, 229, 427-445. [CrossRef]

28. Stich, K.; Eidenberger, T.; Wurst, F. Flavonol synthase activity and the regulation of flavonol and anthocyanin biosynthesis during flower development in Dianthus caryophyllus L. (carnation). Z. Naturforsch. 1992, 47, 553-560. [CrossRef]

29. Mori, S.; Asano, S.; Kobayashi, H.; Nakano, M. Analyses of anthocyanidins and anthocyanins in flowers of Muscari spp. Ngt. Dgk. Ngk. Knkyu. Hkk. 2002, 55, 13-18.

30. Yoshida, K.; Aoki, H.; Kameda, K.; Kondo, T. Structure of muscarinin A, an acylated anthocyanin, from purplish blue spicate flower petals of Muscari arumeniacum. ITE Lett. Batter New Technol. Med. 2002, 1, 35-38.

31. Qi, Y.; Lou, Q.; Li, H.; Yue, J.; Liu, Y.; Wang, Y. Anatomical and biochemical studies of bicolored flower development in Muscari latifolium. Protoplasma 2013, 250, 1273-1281. [CrossRef]

32. Markham, K.; Ofman, D. Lisianthus flavonoid pigments and factors influencing their expression in flower colour. Phytochemistry 1993, 34, 679-685. [CrossRef]

33. Gronquist, M.; Bezzerides, A.; Attygalle, A.; Meinwald, J.; Eisner, M.; Eisner, T. Attractive and defensive functions of the ultraviolet pigments of a flower (Hypericum calycinum). Proc. Natl. Acad. Sci. USA 2001, 98, 13745-13750. [CrossRef]

34. Mo, Y.; Nagel, C.; Taylor, L. Biochemical complementation of chalcone synthase mutants defines a role for flavonols in functional pollen. Proc. Natl. Acad. Sci. USA 1992, 89, 7213-7217. [CrossRef] [PubMed] 
35. Kuhn, B.; Geisler, M.; Bigler, L.; Ring, C. Flavonols accumulate asymmetrically and affect auxin transport in Arabidopsis. Plant Physiol. 2011, 156, 585-595. [CrossRef] [PubMed]

36. Saslowsky, D.; Warek, U.; Winkel, B. Nuclear localization of flavonoid enzymes in Arabidopsis. J. Biol. Chem. 2005, 280, 23735-23740. [CrossRef]

37. Yoo, S.; Cho, Y.; Sheen, J. Arabidopsis mesophyll protoplasts: a versatile cell system for transient gene expression analysis. Nat. Protoc. 2007, 2, 1565-1572. [CrossRef]

38. Horsch, R.; Fry, J.; Hoffmann, N.; Eichholtz, D.; Rogers, S.; Fraley, R. A simple and general method for transferring genes into plants. Science 1985, 227, 1229-1232.

39. Gao, L.; Mazza, G. Characterization, quantitation, and distribution of anthocyanins and colorless phenolics in sweet cherries. J. Agric. Food Chem. 1995, 43, 343-346. [CrossRef]

40. Hashimoto, F.; Tanaka, M.; Maeda, H.; Shimizu, K.; Sakata, Y. Characterization of cyanic flower color of Delphinium cultivars. J. Jpn. Soc. Hort. Sci. 2000, 69, 428-434. [CrossRef]

41. Zhang, X.; Shi, Q.; Ji, D.; Niu, L.; Zhang, Y. Determination of the phenolic content, profile, and antioxidant activity of seeds from nine tree peony (Paeonia section Moutan DC.) species native to China. Food Res. Int. 2017, 97, 141-148. [CrossRef] [PubMed]

Sample Availability: Samples of the compounds analyzed in the study are unavailable from the authors due to their isolation on a small scale.

(C) 2019 by the authors. Licensee MDPI, Basel, Switzerland. This article is an open access article distributed under the terms and conditions of the Creative Commons Attribution (CC BY) license (http://creativecommons.org/licenses/by/4.0/). 